\title{
Large amplitude oscillatory motion along a solar filament ${ }^{\star}$
}

\author{
B. Vršnak ${ }^{1}$, A. M. Veronig ${ }^{2}$, J. K. Thalmann ${ }^{3}$, and T. Žic ${ }^{1}$ \\ 1 Hvar Observatory, Faculty of Geodesy, Kačićeva 26, 10000 Zagreb, Croatia \\ e-mail: bvrsnak@geof.hr \\ 2 Institute of Physics, University of Graz, Universitätsplatz 5, $8010 \mathrm{Graz}$, Austria \\ 3 Max-Planck-Institut für Sonnensystemforschung, Max-Planck-Str. 2, 37191 Katlenburg-Lindau, Germany
}

Received 18 April 2007 / Accepted 22 May 2007

ABSTRACT

\begin{abstract}
Context. Large amplitude oscillations of solar filaments is a phenomenon that has been known for more than half a century. Recently, a new mode of oscillations, characterized by periodical plasma motions along the filament axis, was discovered.

Aims. We analyze such an event, recorded on 23 January 2002 in Big Bear Solar Observatory H $\alpha$ filtergrams, to infer the triggering mechanism and the nature of the restoring force.

Methods. Motion along the filament axis of a distinct buldge-like feature was traced, to quantify the kinematics of the oscillatory motion. The data were fitted by a damped sine function to estimate the basic parameters of the oscillations. To identify the triggering mechanism, morphological changes in the vicinity of the filament were analyzed.

Results. The observed oscillations of the plasma along the filament were characterized by an initial displacement of $24 \mathrm{Mm}$, an initial velocity amplitude of $51 \mathrm{~km} \mathrm{~s}^{-1}$, a period of $50 \mathrm{~min}$, and a damping time of $115 \mathrm{~min}$. We interpret the trigger in terms of poloidal magnetic flux injection by magnetic reconnection at one of the filament legs. The restoring force is caused by the magnetic pressure gradient along the filament axis. The period of oscillations, derived from the linearized equation of motion (harmonic oscillator) can be expressed as $P=\pi \sqrt{2} L / v_{\mathrm{A} \varphi} \approx 4.4 L / v_{\mathrm{A} \varphi}$, where $v_{\mathrm{A} \varphi}=B_{\varphi 0} / \sqrt{\mu_{0} \rho}$ represents the Alfvén speed based on the equilibrium poloidal field $B_{\varphi 0}$.

Conclusions. Combination of our measurements with some previous observations of the same kind of oscillations shows good agreement with the proposed interpretation.
\end{abstract}

Key words. Sun: filaments - magnetohydrodynamics (MHD)

\section{Introduction}

Solar coronal structures are frequently subject to oscillations of various modes and time/spatial scales (e.g., Aschwanden 2003). The longest known phenomenon of this kind are so called winking filaments (Ramsey \& Smith 1966). They represent large-amplitude large-scale oscillations of prominences observed on the solar disc, most often triggered by disturbances coming from distant flares. Various modalities of prominence oscillatory motions were subsequently reported (Kleczek \& Kuperus 1969; Malville \& Schindler 1981; Vršnak 1984; Wiehr et al. 1984; Tsubaki \& Takenchi 1986; Vršnak et al. 1990; Jing et al. 2003; Isobe \& Tripathi 2006). The phenomenon is generally interpreted in terms of different magnetohydrodynamical (MHD) wave modes (for a classification refer to reviews by Tsubaki 1988; Vršnak 1993; Roberts 2000; Oliver \& Ballester 2002).

Most of reported large-amplitude large-scale oscillations occur perpendicular to the prominence axis. The restoring force in this type of oscillation generally can be explained in terms of the magnetic tension (Hyder 1966; Kleczek \& Kuperus 1969; Vršnak 1984; Vršnak et al. 1990). On the other hand, the oscillation damping in the corona is attributed either to the "aerodynamic" drag (i.e., the energy loss by emission of waves into the ambient corona; Kleczek \& Kuperus 1969), or to various dissipative processes (Hyder 1966; Nakariakov et al. 1999;

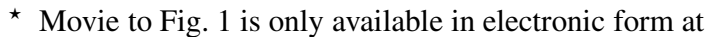
http://www . aanda. org
Terradas et al. 2001; Ofman \& Aschwanden 2002; Verwichte et al. 2004).

Recently, Jing et al. (2003, 2006) reported a new mode of oscillations in filaments/prominences, where the oscillatory motion occurs along the prominence axis. In this paper we report another example observed in $\mathrm{H} \alpha$ filtergrams and propose an explanation for the triggering process and the restoring force.

\section{Observations}

The filament that we discuss was located near the center of the solar disk on 2002 January 23 and was observed in fulldisk $\mathrm{H} \alpha$ filtergrams at $1^{\prime \prime}$ /pixel resolution with a time cadence of $1 \mathrm{~min}$ at the Big Bear Solar Observatory (BBSO) Global $\mathrm{H} \alpha$ Network Station (Steinegger et al. 2000). The accompanying movie shows the evolution of the oscillating filament between 17 and 22 UT; snapshots are presented in Fig. 1. During this time span, the filament was activated and showed oscillations along its main axis which could be followed for 5 cycles.

Before the measurements, we co-aligned all the images to the reference image taken at 17:00 UT (hereinafter used as $t=0$ ), correcting for solar differential rotation and also for image jittering using cross-correlation techniques. For measuring the oscillatory motions of the filament, we did not use all the available images but on average every third image of the series, giving an average time cadence of 3 min.

The main part of the filament, 110-120 Mm long, was following the straight magnetic inversion line oriented in 


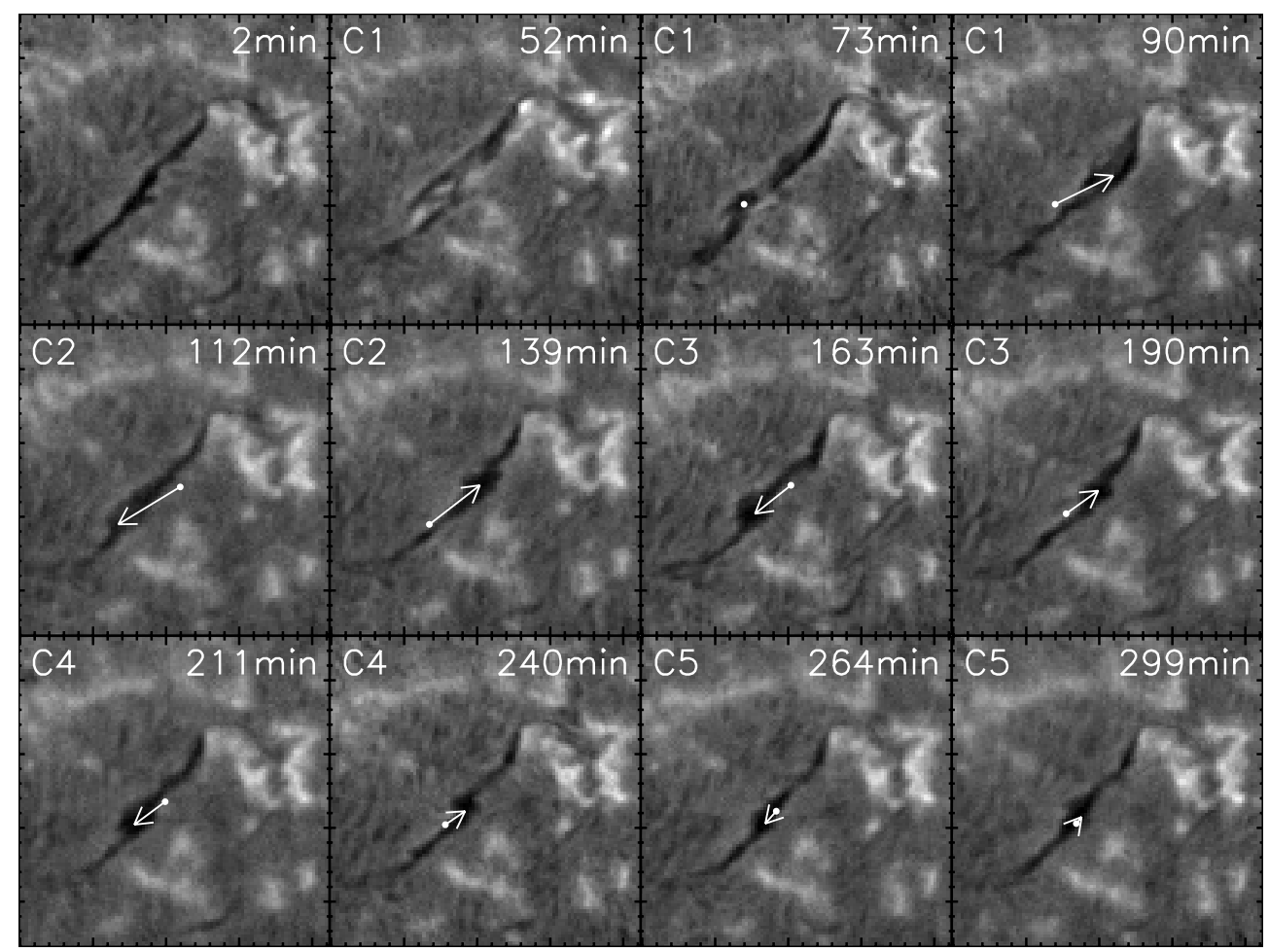

Fig. 1. Sequence of BBSO H $\alpha$ images for a $210^{\prime \prime} \times 210^{\prime \prime}$ subfield. For each image, the observation time (in minutes after 17:00 UT) is annotated. The arrows connect the determined displacement for consecutive images taken around the oscillation peaks for each of the five cycles (C1-C5). The arrowhead indicates the determined center of the followed filament feature in the actual image, the circle drawn at the other end of the arrow indicates the location of the same feature as determined from the previous image shown in the figure. Thus, the decreasing lengths of the arrows directly reflect the instantaneous oscillation amplitudes and their damping. See also the accompanying movie.
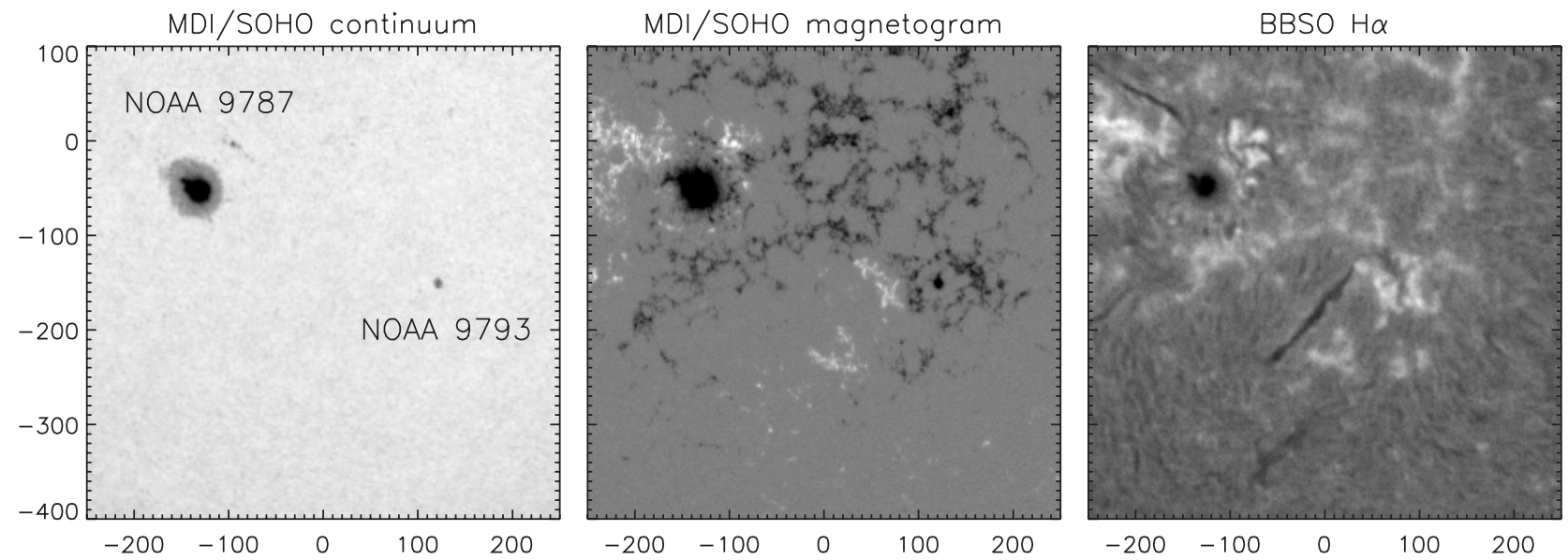

Fig. 2. MDI/SOHO continuum image, MDI/SOHO magnetogram and BBSO $\mathrm{H} \alpha$ filtergram at 17 UT of active regions NOAA 9787 and NOAA 9793 with the filament. Units of the $x$ - and $y$-axis are in arc sec.

southeast-northwest direction. Its northwest leg, a curved thread of length 20-30 Mm was rooted in a small active region, NOAA 9793 (Fig. 2). The southeast leg was rooted in the quiet chromosphere. For the total length of the filament we take $2 L=$ $140 \mathrm{Mm}$.

The kinematics of the observed oscillations was determined by visually inspecting the $\mathrm{H} \alpha$ image sequence, and measuring the displacement along the filament axis of the most prominent (dark) bulge-like feature in the filament (Fig. 1; see also the accompanying movie). The measured displacements are presented in Fig. 3a, and the corresponding velocities in Fig. 3b. Note that plasma motions could be seen all along the filament, with the amplitude decreasing towards the filament legs, i.e., the oscillation amplitude was largest in the middle part of the filament, where the bulge-like feature was located. A mechanical analogue of the observed oscillation would be, e.g., a longitudinal-mode standing wave on a slinky spring fixed at both ends.

It seems that the filament oscillation was a response to an energy release in the active region, where a weak flare-like brightening appeared in $\mathrm{H} \alpha$ at 17:33 UT in the region of the northwest footpoint of the filament (see the second panel in the first row of Fig. 1; see also the accompanying movie). The $\mathrm{H} \alpha$ subflare achieved a maximum around 17:45 UT, as can be seen in Fig. 3c where we show the $\mathrm{H} \alpha$ light curve, measured at the western kernel of the subflare (see the inset in Fig. 3c; the eastern kernel was 

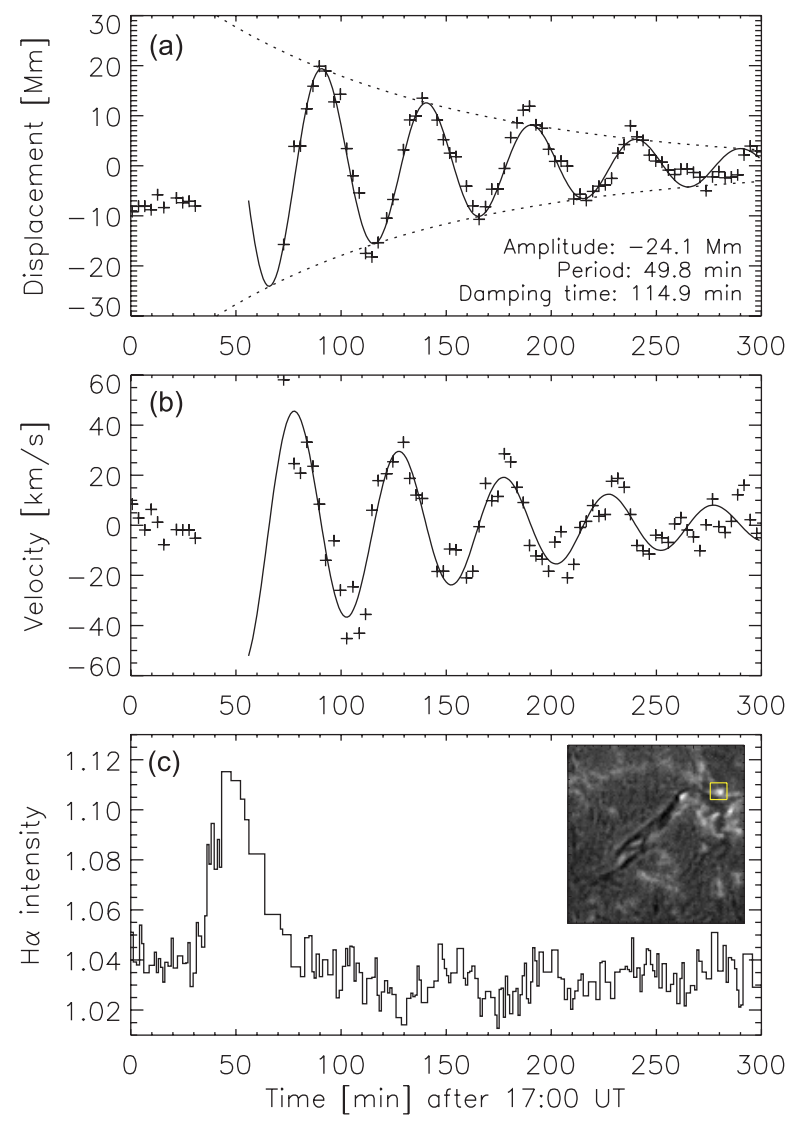

Fig. 3. a) Displacement measurements of the oscillating filament together with the applied fit. b) Velocities derived via numerical differentiation from the displacement measurements (for the procedure see main text) shown together with the time-derivative of the $d(t)$ fit shown in panel a). c) $\mathrm{H} \alpha$ light curve measured in the western footpoint of the associated subflare indicated by the box in the inserted image.

partly obscured by the activated filament, thus it was not possible to measure the lightcurve in this part of the subflare. The energy release associated with the $\mathrm{H} \alpha$ brightening was obviously weak, since no increase in the GOES full-disk integrated soft X-ray flux was observed.

The subflare caused the filament activation, where various forms of motions and restructuring could be observed. Due to complex motions and morphological changes of the filament, in the period 17:35-18:10 it was practically impossible to identify, and measure reliably, the position of the filament feature which was later used to trace the oscillations. In the period between 18:00 and 18:10 UT the motions gradually became more ordered, mainly showing a flow of the filament plasma in the southeast direction, away from the active region. After this initial plasma displacement, the first northwest directed swing started around 18:10 UT (Fig. 3a; the first oscillation measurement was performed at 08:12 UT).

The measured displacement curve, $d(t)$, of the oscillatory filament motion was fitted by a damped sine function of the form

$\tilde{d}(t)=x_{0} \sin \left(\frac{2 \pi t}{P}+\varphi\right) \exp \left(-\frac{t}{\tau}\right)+d_{0}$

with amplitude $x_{0}$, period $P$, phase $\varphi$, damping time $\tau$ and equilibrium position $d_{0}$. Figure 3 a shows the observed oscillatory motion of the filament (with $d_{0}$ already subtracted), together with the fitted curve. In Fig. $3 b$ we plot the corresponding velocities obtained by numerical differentiation of the displacement data points (applying a 3-point, Lagrangian interpolation) together with the time derivative of the $d(t)$ fit.

The basic parameters of the oscillations are:

- period $P=49.8 \mathrm{~min}$;

- initial amplitude $x_{0}=24.1 \mathrm{Mm}$;

- initial velocity amplitude $v_{0}=51 \mathrm{~km} \mathrm{~s}^{-1}$;

- damping time $\tau=114.9 \mathrm{~min}$.

\section{Interpretation and discussion}

The oscillations described by Eq. (1) are the solution of the equation of motion of the form:

$\ddot{x}+2 \delta \dot{x}+\omega_{0}^{2} x=0$.

The cyclic frequency of oscillations $\omega$ is related to the frequency of free oscillations $\omega_{0}$ and the damping rate $\delta$ by the expression $\omega^{2}=\omega_{0}^{2}-\delta^{2}$. From the period of oscillations and the damping time determined in Sect. 2 one finds the cyclic frequency of oscillations and the damping rate as $\omega=2 \pi / P=2.1 \times 10^{-3} \mathrm{~s}^{-1}$ and $\delta=1 / \tau=1.5 \times 10^{-4} \mathrm{~s}^{-1}$, respectively. Since $\omega \gg \delta$, it can be concluded that $\omega_{0} \approx \omega$, so in the following we take for the eigenmode frequency of the system the value $\omega_{0} \approx 2 \times 10^{-3} \mathrm{~s}^{-1}$. From the cyclic frequency $\omega_{0}$ and the amplitude $x_{0}$ one finds the initial velocity and acceleration amplitudes, $v_{0}=\omega_{0} x_{0}=50.8 \mathrm{~km} \mathrm{~s}^{-1}$ and $a_{0}=\omega_{0}^{2} x_{0}=107 \mathrm{~m} \mathrm{~s}^{-2}$.

The estimated value of acceleration gives a constraint to the nature of the restoring force. Let us first assume that the restoring force is governed by the gas pressure gradient. It can be approximately estimated as $\Delta p / 2 L$, where we assume that there is a pressure difference $\Delta p$ over the filament length $2 L$. Such a pressure gradient would give rise to an acceleration of $a=\Delta p / 2 L \rho$, where $\rho$ is the prominence density. Using an order of magnitude estimate $2 L a=\Delta p / \rho \approx p / \rho \approx c_{\mathrm{s}}^{2}$, one finds that the sound speed has to be $c_{\mathrm{s}}=\sqrt{2 L a} \gtrsim 100 \mathrm{~km} \mathrm{~s}^{-1}$. Such a sound speed requires temperatures $\gtrsim 10^{6} \mathrm{~K}$, which is typical for the coronal plasma. So, the required sound speed is an order of magnitude too high, since the temperature of the prominence plasma is $\approx 10^{4} \mathrm{~K}$, corresponding to a sound speed in the order of $c_{\mathrm{s}} \approx 10 \mathrm{~km} \mathrm{~s}^{-1}$. A similar conclusion could be drawn by taking into account that the period of oscillation can be roughly identified with the sound wave travel time along the filament axis, $t_{\mathrm{s}} \approx 2 L / c_{\mathrm{s}}$, i.e., expressing the period as $P \approx 2 t_{\mathrm{s}}=4 L / c_{\mathrm{s}}$. Substituting $2 L=140 \mathrm{Mm}$, we find again $c_{\mathrm{s}}>100 \mathrm{~km} \mathrm{~s}^{-1}$ (note that using the oscillation amplitude $x_{0}$ instead of the filament length $2 L$ would give an estimate of the velocity amplitude and not the oscillation-related wave velocity, i.e., the sound speed).

Thus, the pressure pulse scenario would be possible only if the pressure of the filament plasma was increased by a factor of 100 . That corresponds to a temperature increase from $T_{0} \approx 10^{4} \mathrm{~K}$ to $T \gtrsim 10^{6} \mathrm{~K}$, but no signature of such plasma heating was observed in the TRACE $171 \AA(T \sim 1 \mathrm{MK})$ EUV images. Another possibility is that the restoring force is of magnetic origin. For example, let us consider that the filament is embedded in a flux rope and that at a specific moment an additional poloidal flux is injected into the rope at one of its legs. The flux could be injected from below the photosphere, or by magnetic field reconnection (Uchida et al. 2001; Jibben \& Canfield 2004). Indeed, $\mathrm{H} \alpha$ flare-like brightenings appeared just before or around the onset of oscillations in the region of the northwest footpoint of the filament (see Sect. 2), which is likely to be a signature of reconnection. On the other hand, no sign of the emerging flux process could be detected in the region around the 
filament in the SOHO/MDI magnetograms which were available at a time cadence of $1 \mathrm{~min}$.

The excess poloidal field creates a magnetic pressure gradient and a torque, driving a combined axial and rotational motion of plasma (Jockers 1978; Uchida et al. 2001). If the injection has a shorter duration than the Alfvén travel time along the flux rope axis, it causes a perturbation that propagates along the rope (Uchida et al. 2001) and evolves into an oscillatory motion along its axis. Here we assume that the wave caused by the injection is reflected at the opposite footpoint of the rope, and that the damping rate is smaller than the oscillation cyclic frequency. Also note that since $c_{\mathrm{S}}<v_{\mathrm{A}}$ the plasma is carried by $B_{\varphi}$ since plasma motions along the field lines are slower than translation of poloidal field (fast-mode MHD wave).

Consider a flux rope of a constant width where a certain amount of poloidal flux is injected at one of its footpoints (similar to Fig. 5 of Jibben \& Canfield 2004), over an interval shorter than the eigenmode period of the related oscillation mode. We divide the flux rope in equilibrium into two segments of length $L$, symmetric with respect to the flux rope midpoint. In the simplest form, the average poloidal magnetic field in the two segments, after perturbing the flux rope, can be expressed as $B_{\varphi 1}=B_{\varphi 0} L /(L-x)$ and $B_{\varphi 2}=B_{\varphi 0} L /(L+x)$. Here, $B_{\varphi 0}$ is the poloidal field in the equilibrium state and $x$ is the longitudinal displacement of the plasma element separating the two segments (in the equilibrium taken to be at the filament barycenter, located at the midpoint of the flux rope). We use these relations to express the corresponding magnetic pressures, $p_{m}=B_{\varphi}^{2} / 2 \mu_{0}$, which we substitute into the simplified equation of motion:

$\rho \ddot{x} \approx-\frac{\Delta p_{m}}{L}=-\frac{B_{\varphi 0}^{2}}{2 \mu_{0}}\left[\frac{1}{(L-x)^{2}}-\frac{1}{(L+x)^{2}}\right] L$,

where $\Delta p_{m} / L$ represents approximately the gradient of the poloidal-field magnetic pressure. After linearizing Eq. (3), and expressing the displacement in the dimensionless form $X=x / L$, we get the equation of motion of the harmonic oscillator:

$\ddot{X}=-\frac{2 v_{\mathrm{A} \varphi}^{2}}{L^{2}} X$,

where $v_{\mathrm{A} \varphi}=B_{\varphi 0} / \sqrt{\mu_{0} \rho}$ represents the Alfvén speed based on the equilibrium poloidal field $B_{\varphi 0}$.

Equation (4) implies $\omega_{0}^{2}=2 v_{\mathrm{A} \varphi}^{2} / L^{2}$, and consequently, $P=\pi \sqrt{2} L / v_{\mathrm{A} \varphi} \approx 4.4 L / v_{\mathrm{A} \varphi}$. Using the observed parameters, we find $v_{\mathrm{A} \varphi} \approx 100 \mathrm{~km} \mathrm{~s}^{-1}$. Assuming that the number density of the prominence plasma is in the range $n=10^{10}-10^{11} \mathrm{~cm}^{-3}$ (cf. Tandberg-Hanssen 1995), one finds $B_{\varphi 0} \approx 5-15$ Gauss.

In this respect we note the filament was composed of helicallike fine structure patterns, twisted around the filament axis. Bearing in mind that the frozen-in condition is satisfied in the prominence plasma, such patterns are indicative of the fluxrope helical magnetic field (Vršnak et al. 1991). In the period 18:25-18:40 UT the internal structure was seen clearly enough to provide measurements of the pitch angle of helical threads. The measured values of the pitch angle range between $20^{\circ}$ and $30^{\circ}$, with the mean value $\tan \vartheta=24^{\circ} \pm 4^{\circ}$. Bearing in mind $\tan \vartheta=B_{\varphi} / B_{\|}$, where $B_{\varphi}$ and $B_{\|}$are the poloidal and axial magnetic field components (Vršnak et al. 1991), and considering the poloidal field of 5-15 Gauss, one finds $B_{\|} \approx 10-30$ Gauss, which is a reasonable value for quiescent prominences (Tandberg-Hanssen 1995).

According to Vršnak (1990), who treated the stability of a semitoroidal flux rope anchored at both legs in the photosphere, the prominence with pitch angle $\vartheta=20^{\circ}-30^{\circ}$ should be stable, which is consistent with the fact that the filament was severely activated, but did not erupt. A similar conclusion can be drawn if the total number of turns of the helical field line is considered. Taking into account that the filament width was around $2 r \approx 2$ arcsec and employing the expression for the pitch-length $\lambda=2 \pi r / \tan \vartheta$, we find the total number of turns of the helical field line $N=2 L / \lambda \approx 1.4$. This is again consistent with the model stability-criteria by Vršnak 1990 (for the unstable cases of highly twisted quiescent prominences see, e.g., Vršnak et al. 1991; Karlický \& Šimberová 2002). However, it should be noted that sometimes active region prominences erupt at smaller number of turns $(N \gtrsim 1$; e.g., Rust \& Kumar 1996; Régnier \& Amari 2004; Salman et al. 2007).

Regarding the $P \propto L$ relationship, Fig. 5a of Jing et al. (2006) must be considered, where in the figure caption it is stated that the $x$-axis of the graph represents the "length". However, checking Table 1 therein, one finds that in fact the $x$-axis represents the oscillation amplitude, which we previously denoted as $x_{0}$. Consequently, the lack of correlation does not really mean that there is no $P \propto L$ relationship. In this respect it is instructive to comment also Fig. 5b of Jing et al. (2006) which shows proportionality of the velocity amplitude $v_{0}$ and "length", the latter in fact representing the amplitude $x_{0}$. Such a proportionality is expected for any harmonic oscillator since $v_{0}=\omega_{0} x_{0}$. Bearing in mind Eq. (4), i.e., $\omega_{0} \propto 1 / L$, one would expect an inverse proportionality between $v_{0}$ and $L$ in the case of equal amplitudes.

Out of four events studied by Jing et al. (2003, 2006), in two cases it is possible to estimate the overall length of the oscillating filaments. From filtergrams displayed in Fig. 1 of Jing et al. (2003) and Fig. 3 of Jing et al. (2006), showing filaments characterized by periods $P=80$ and $100 \mathrm{~min}$, we estimated the filament lengths to approximately $2 L \approx 200 \mathrm{Mm}$ and $2 L \approx 300 \mathrm{Mm}$, respectively. Combining these values with our measurements $(P=50 \mathrm{~min} ; 2 L \approx 140 \mathrm{Mm})$, we find that the data follow approximately the $P \propto L$ dependence. The linear least squares fit gives a slope of $0.6 \mathrm{~min} / \mathrm{Mm}$, or $0.7 \mathrm{~min} / \mathrm{Mm}$ if the fit is fixed at the origin. If we compare these values with the previously derived relationship $P \approx 4.4 L / v_{\mathrm{A} \varphi}$, we find that these slopes correspond to $v_{\mathrm{A} \varphi} \approx 100-120 \mathrm{~km} \mathrm{~s}^{-1}$, which is in agreement with the value of $v_{\mathrm{A} \varphi}$ that we estimated earlier for our oscillating filament.

Finally, let us note that the poloidal flux injection changes the poloidal-to-axial field ratio $B_{\varphi} / B_{\|}$, which should also cause oscillations perpendicular to the prominence axis, since the equilibrium height of the prominence is directly related to ratio $B_{\varphi} / B_{\|}$(Vršnak 1984, 1990). Indeed, careful inspection of the filament motion reveals such transversal oscillations of a small amplitude, synchronous with the longitudinal ones (see the accompanying movie). The analysis of the interplay between the longitudinal, transversal, torsional (pitch-angle), and radial oscillations requires meticulous consideration, which will be presented in a separate paper.

\section{Conclusion}

The observed oscillations of the plasma along the filament were characterized by a period of $50 \mathrm{~min}$, velocity amplitude of $50 \mathrm{~km} \mathrm{~s}^{-1}$, and damping time of $115 \mathrm{~min}$. Our analysis indicates that the oscillations of the filament plasma along its axis were driven magnetically as in other types of prominence oscillations. We propose that the oscillations were triggered by an injection of poloidal magnetic field into the flux rope, most probably by the reconnection associated with a subflare that took place at the 


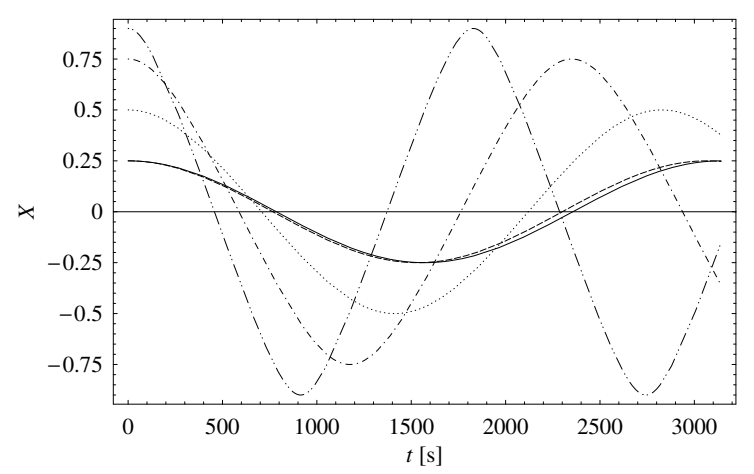

Fig. A.1. Solutions of Eq. (5) shown for amplitudes $X_{0}=0.25,0.5$, 0.75 , and 0.9 (dashed, dotted, dot-dashed and dot-dot-dashed line respectively), together with the harmonic oscillator solution for $X_{0}=0.25$ (full line).

northwest leg of the filament. The flux injection is expected to cause a translatory and torsional motion of plasma, propagating toward the other leg of the filament. After the reflection of the wave, the oscillations along the filament axis develop, showing an order of magnitude weaker damping than in perpendicular oscillations of filaments (for the latter see, e.g., Hyder 1966). Most probably, perpendicular oscillations decay much faster because they affect the ambient corona more than motions along the flux tube, i.e., the energy flux carried away by MHD waves is much larger.

The period in our event is shorter than in analogous oscillations analyzed by Jing et al. (2003, 2006), where the periods were in the range from 80 to $160 \mathrm{~min}$. In two of these events the filament length could be measured, and when supplemented with our event, we find that the periods follow the $P \propto L$ relationship. The slope of the relationship is consistent with a poloidal magnetic field in the order of 10 Gauss.

The damping time $\tau$ is also shorter than in the events analyzed by Jing et al. (2003, 2006). If our measurements were inserted into Fig. 5c of Jing et al. (2006), the corresponding datapoint would be located very close the lower end of the regression line presented therein. The linear least squares fit, with the axis intercept fixed at the origin, would read $\tau=3.5 P$.

\section{Appendix: The nonlinearity aspect}

Since the filament oscillations were characterized by large amplitudes, it is instructive to check how much the harmonicoscillator approximation differs from the solutions of Eq. (3). After introducing the dimensionless displacement $X=x / L$, Eq. (3) can be rewritten in the form:

$\ddot{X}=-\frac{\omega_{0}^{2} X}{\left(1-X^{2}\right)^{2}}$,

where $\omega_{0}^{2}=2 v_{\mathrm{A} \varphi}^{2} / L^{2}$. In Fig. A.1 we show the solutions of Eq. (5) for amplitudes $X_{0}=0.25,0.5,0.75$, and 0.9 together with the harmonic oscillator solution for $X_{0}=0.25$. We have chosen $\omega_{0}^{2}=4 \times 10^{-6} \mathrm{~s}^{-1}$, approximately corresponding to $P=50 \mathrm{~min}$. Inspecting Fig. A.1 one finds that the period decreases with increasing amplitude: it is about $2 \%$ shorter than the harmonic oscillator period for $X_{0}=0.25$, around $10 \%$ shorter at $X_{0}=0.5$, and $25 \%$ at $X_{0}=0.75$. The deviation from the harmonic oscillator period at $X_{0}=0.9$ becomes larger than $40 \%$. Bearing in mind the damping, the increase of the period with decreasing amplitude means that the period should increase as the oscillations attenuate. Indeed, going back to Fig. 3, we find that the measurements might indicate such a trend towards the end of oscillations, in particular after $t>250 \mathrm{~min}$.

Acknowledgements. We are thankful to Vasyl Yurchyshyn for providing us with the data from the Global High Resolution $\mathrm{H} \alpha$ Network, operated by the Big Bear Solar Observatory, New Jersey Institute of Technology. We are grateful to the referee, Dr. Marian Karlický, whose constructive suggestions led to significant improvements in this paper. Travel grants from the exchange program AustriaCroatia WTZ 08/2006 are gratefully acknowledged.

\section{References}

Ali, S. S., Uddin, W., Chandra, R., Mary, D. L., \& Vršnak, B. 2007, Sol. Phys., 240, 89

Aschwanden, M. J. 2003, in Turbulence, Waves, and Instabilities in the Solar Plasma, ed. R. von Fay-Siebenburgen, K. Petrovay, B. Roberts, \& M. J. Aschwanden, NATO Science Ser. (Kluwer Academic Publishers), 215

Hyder, C. L. 1966, ZAp, 63, 78

Isobe, H., \& Tripathi, D. 2006, A\&A, 449, L17

Jibben, P., \& Canfield, C. 2004, ApJ, 610, 1129

Jing, J., Lee, J., Spirock, T. J., et al. 2003, ApJ, 584, L103

Jing, J., Lee, J., Spirock, T. J., \& Wang, H. 2006, Sol. Phys., 236, 97

Jockers, K. 1978, ApJ, 220, 1133

Karlický, M., \& Šimberová, S. 2002, A\&A, 388, 1016

Kleczek, J., \& Kuperus, M. 1969, Sol. Phys., 6, 72

Malville, J. M., \& Schindler, M. 1981, Sol. Phys., 70, 115

Nakariakov, V. M., Ofman, L., DeLuca, E., Roberts, B., \& Davila, J. M. 1999, Science, 285, 862

Ofman, L., \& Aschwanden, M. J. 2002, ApJ, 576, L153

Oliver, R., \& Ballester, J. L. 2002, Sol. Phys., 206, 45

Ramsey, H. E., \& Smith, S. F. 1966, AJ, 71, 197

Rust, D. M., \& Kumar, A. 1996, ApJ, 464, L199

Régnier, S., \& Amari, T. 2004, A\&A, 425, 345

Roberts, B. 2000, Sol. Phys., 193, 139

Steinegger, M., Denker, C., Goode, P. R., et al. 2000, Proceedings of the 1st Solar and Space Weather Euroconference, ed. A. Wilson, ESA SP-463, 617

Tandberg-Hanssen, E. 1995, The Nature of Solar Prominences (Dordrecht: Kluwer)

Terradas, J., Oliver, R., \& Ballester, J. L. 2001, A\&A, 378, 635

Tsubaki, T. 1988, in Solar and Stellar Coronal Structures and Dynamics, ed. R. C. Altrock, National Solar Observatory, 140, Fluid Dynam., 3, 89

Tsubaki, T., \& Takenchi, A. 1986, Sol. Phys., 104, 313

Uchida, Y., Yabiku, T., Cable, S., \& Hirose, S. 2001, PASJ, 53, 331

Verwichte, E., Nakariakov, V. M., Ofman, L., \& Deluca, E. E. 2004

Vršnak, B. 1984, Sol. Phys., 94, 289

Vršnak, B. 1990, Sol. Phys., 129, 295

Vršnak, B. 1993, Hvar Obs. Bull., 202, 173

Vršnak, B., Ruždjak, V., Brajša, R., \& Zloch, F. 1990, Sol. Phys., 127, 119

Vršnak, B., Ruždjak, V., \& Rompolt, B. 1991, Sol. Phys., 136, 151

Wiehr, E., Stellmacher, G., \& Balthasar, H. 1984, Sol. Phys., 94, 285 